197. W. Rindt, M. Konstandin, P. Altmayer, A. Weigerding (Homburg/Saar): Serum und Tumorkonzentrationen eines neuen Podophyllotoxinderivates

Die Wirkung eines Zytostatikums ist charakterisiert durch die Tumorgenerationszeiten, die Synchronisation derselben, die Pharmakokinetik der Substanz, seine Tumorgängigkeit und durch die maximale Konzentration, die mit dem hämopoetischen System noch kompatibel ist. In diesem Zusammenhang wurden Pharmakokinetik und Gewebskonzentrationen eines neuen Podophyllotoxinderivates (4-Demethylepipodophyllotoxin- $\beta$-D-äthylidenglucosid) an sechs $\mathrm{Pa}$ tientinnen mit gynäkologischen Malignomen (Mamma-, Korpus- und Ovarialkarzinom) untersucht. Die Substanz war tritiummarkiert und wurde i.v. appliziert. Die Serumkonzentrationen wurden einem Iterationsprogramm unterworfen; folgende pharmakokinetischen Konstanten wurden ermittelt: bei einem Korrelationskoeffizienten von 0,988 und einer Restvarianz von $2,23 \%$ betrug die globale Eliminatinskonstante 1,376 $\pm 0,532$. Die Verteilungshalbwertszeit war $0,124 \pm 0,059$, die Eliminationshalbwertszeit 3,234 $\pm 1,628$. Das Verteilungsvolumen betrug zentral $11,05 \pm 3,404$, im "steady-state" $64,62 \pm 58,3$ l. Die totale Clearance war $15,03 \pm 6,493 \mathrm{l} / \mathrm{h}$. Die „mean-transit-time“ betrug zentral $0,824 \pm 0,32 \mathrm{~h}$, im ,steady-state“ $3,867 \pm 1,905 \mathrm{~h}$.

Die Gewebskonzentrationen beliefen sich auf $2,74 \mathrm{pMol} / \mathrm{g}$ bei einer Metastase eines Korpuskarzinoms, 1,39-5,4 bei Mammakarzinom, 3,36 in Lymphknoten, 4,38 in der Haut und 0,27-0,66 $\mathrm{pMol} / \mathrm{g}$ im Fettgewebe.

Im Vergleich $\mathrm{zu}$ vielen anderen Zytostatika ist das geprüfte Präparat charakterisiert durch rasche Verteilung und Elimination. Insofern könnte es sich eignen für eine Stoßtherapie nach Synchronisation.

K. Hamada, T. Tamura, T. Sugawa (Osaka): Behandlungsergebnisse bei 210 Trophoblasttumoren

Es ist eine bekannte Tatsache, daß Zytostatika bei Trophoblastgeschwülsten im Gegensatz zu anderen malignen Tumoren gut wirksam sind. Ebenso wissen wir, daß histologisch gesicherte Chorionkarzinome auch heute noch eine hohe Todesrate aufweisen. In unserem Bemühen, die Prognose von Trophoblastgeschwulsten zu verbessern, haben wir aus unserem Krankengut speziell die schweren Fälle selektiert, sie nach bestimmten Kriterien zwei Untergruppen zugeordnet und entsprechend unserem Therapieschema behandelt. Im folgenden berichten wir über die Ergebnisse unserer Behandlung an insgesamt 210 Patientinnen mit Trophoblastgeschwülsten aus den vergangenen 8 Jahren.

\title{
Material und Methodik
}

Seit 1972-1979 sahen wir insgesamt 210 Patientinnen mit Trophoblastgeschwülsten, davon waren 140 Fälle mit Blasenmolen, 14 histologisch gesicherte destruierende Blasenmolen, 18 Chorionkarzinome und 38 sogenannte ,fragliche Fälle". Bedingt durch die heute verbesserten Diagnosemöglichkeiten sowie durch den Einsatz wirksamer Zytostatika finden wir mehr sogenannte ,fragliche Fälle“, bei denen eine histologische Absicherung der Diagnose schwer ist. 